\title{
Multi-hop wireless network modelling using OMNET++ simulator
}

\begin{abstract}
The multi-hop networking plays an important role in wireless coverage area and cost reduction. In this paper, we have presented our experience to design the multi-hop wireless network and explained the realistic behavior using OMNET++ simulator. The simulation is based on proper selection of wireless node, routing protocol and other important parameters. This paper presents a detailed analysis about how INET framework can be used to simulate multi-hop wireless network. We also discussed the important modules and methodology to define simulation parameters and analyze the results for the simple scenario of multi-hop wireless network.
\end{abstract}

Keyword: INET; Multi-hop; OMNET++; Simulation; Wireless network 